\title{
Epilepsy in patients with autism: links, risks and treatment challenges
}

This article was published in the following Dove Press journal:

Neuropsychiatric Disease and Treatment

\section{Frank MC Besag}

Neurodevelopmental Team, East London Foundation NHS Trust, Family Consultation Clinic, Bedford, UK

Correspondence: Frank MC Besag Neurodevelopmental Team, East London Foundation NHS Trust, Family Consultation Clinic, 24 Grove Place, Bedford, MK40 3JJ, UK

Email fbesag@aol.com

\begin{abstract}
Autism is more common in people with epilepsy, approximately $20 \%$, and epilepsy is more common in people with autism with reported rates of approximately $20 \%$. However, these figures are likely to be affected by the current broader criteria for autism spectrum disorder (ASD), which have contributed to an increased prevalence of autism, with the result that the rate for ASD in epilepsy is likely to be higher and the figure for epilepsy in ASD is likely to be lower. Some evidence suggests that there are two peaks of epilepsy onset in autism, in infancy and adolescence. The rate of autism in epilepsy is much higher in those with intellectual disability. In conditions such as the Landau-Kleffner syndrome and nonconvulsive status epilepticus, the epilepsy itself may present with autistic features. There is no plausible mechanism for autism causing epilepsy, however. The co-occurrence of autism and epilepsy is almost certainly the result of underlying factors predisposing to both conditions, including both genetic and environmental factors. Conditions such as attention deficit hyperactivity disorder, anxiety and sleep disorders are common in both epilepsy and autism. Epilepsy is generally not a contraindication to treating these conditions with suitable medication, but it is important to take account of relevant drug interactions. One of the greatest challenges in autism is to determine why early childhood regression occurs in perhaps $25 \%$. Further research should focus on finding the cause for such regression. Whether epilepsy plays a role in the regression of a subgroup of children with autism who lose skills remains to be determined.
\end{abstract}

Keywords: epilepsy, autism, regression, genetics, environment, Landau-Kleffner, CSWS

\section{Introduction}

Compared with the general population, the increased prevalence of epilepsy in people with autism and the increased prevalence of autism in epilepsy are now well established. ${ }^{1-3}$ Kanner's ${ }^{4}$ original paper describing 11 children with "autistic disturbances of affective contact" included one child with a history of seizures and an abnormal encephalogram (EEG). Several epidemiologic studies have since confirmed the relatively common co-occurrence of these two conditions. A review by Tuchman and Rapin ${ }^{5}$ stated that the reported frequency of epilepsy in autism ranged from 5\% to about $40 \%$. Amiet et al, ${ }^{1}$ in a meta-analysis of epilepsy in autism, demonstrated a relationship with intellectual disability; epilepsy was present in $21.5 \%$ of subjects with autism who also had intellectual disability and $8 \%$ of subjects without intellectual disability. The relationships between epilepsy and autism continue to be debated. ${ }^{6}$ This relationship is tantalizing from the scientific point of view and challenging from the management point of view. It is anticipated that a greater understanding of the relationship between these two conditions could have profound effects on the management of patients. If the risk factors for developing epilepsy in people with autism were known, perhaps the epilepsy could be prevented, or at least recognized early and 
managed better, as a result. Knowing that certain disorders, for example, metabolic disorders, can lead both to autism and epilepsy should result in early recognition and possible amelioration of the effects of both epilepsy and autism. In previous reviews, ${ }^{6,7}$ the current author had asked a number of questions. These questions remain relevant to the discussion of the relationship between epilepsy and autism. They include the following. Does epilepsy cause autism? Does autism cause epilepsy? Are there underlying brain mechanisms that predispose to both conditions? What is the role of genetics in determining conditions that underlie both autism and epilepsy? What is the importance of prenatal, perinatal and postnatal environmental factors? Do any of the proposed relationships between autism and epilepsy provide insight into useful management or treatment? Is the prognosis of either autism or epilepsy different when the other condition is present? What is the role of additional comorbidities, such as intellectual disability or attention deficit hyperactivity disorder (ADHD), in the relationship between the two conditions and in influencing treatment choices? What research is recommended for the future? Many of the issues reflected in these questions remain controversial. However, a confounding factor that is likely to affect not only the quoted percentages of the co-occurrence of the two conditions, but also the prevalence of additional comorbidities is the major change in the quoted prevalence of autism spectrum disorder (ASD) itself. These issues will be discussed and updated in this review.

\section{The effect of the changing prevalence of ASD}

Early studies, for example, Lotter, ${ }^{8}$ quoted an autism prevalence of about 4 per 10,000. In contrast, recent epidemiologic studies, for example, Baird et al, ${ }^{9}$ and the various estimates of Fombonne and coworkers ${ }^{10,11}$ have indicated that the prevalence of ASD in children is greater than $1 \%$ and the prevalence of Diagnostic and Statistical Manual of Mental Disorders, 5th revision (DSM-5) ASD together with social communication disorder might be $>2 \%$. It is quite clear that the early, narrow diagnostic criteria included only children with more severe autism and, by implication, children with more severe disability overall. The rate of intellectual disability and, consequently, epilepsy would have been much higher in this group of severely affected children than in the much larger group identified by the broader diagnostic criteria in recent studies. However, reviews in the literature have taken little or no account of this major factor. The quoted figures of epilepsy in autism using previous narrow criteria will be much higher than the prevalence using the current criteria. If children with intellectual disability (defined as an intelligence quotient [IQ] <70) are excluded, because even the population of children with autism with IQ $>70$ will be skewed toward a lower-than-average IQ, the prevalence of epilepsy is still likely to be higher than that in the general population. In other words, this increased prevalence of epilepsy in children with autism and IQ $>70$ might be because of the distribution tending toward a lower IQ, rather than because of any specific relationship to autism. In contrast, the broader definition of "ASD" implies that the prevalence of this condition in children with epilepsy might be considerably higher using the current criteria in place of the narrow "Kanner autism" criteria. These issues require a detailed critical review which is beyond the scope of the current overview.

In summary, the previous narrow diagnostic criteria of severe "Kanner" autism were associated with a high rate of intellectual disability and a high rate of epilepsy, whereas the current broader "ASD" criteria would be expected to lead to a lower rate of intellectual disability and a lower rate of epilepsy, but the frequency of ASD in epilepsy would be expected to be higher using the current broader criteria. Most published papers do not take account of the changing ASD criteria, implying that the rates of intellectual disability and epilepsy may be misleading. When quoting such figures, it is important not only to know what diagnostic criteria were used for autism/ASD, but also to note the date of publication because of the increased awareness of autism. The consequence of the changing prevalence of ASD is that past statements about the rate of epilepsy in autism are now almost certainly inaccurate.

\section{At what age does epilepsy develop in children with autism?}

In one of the classic studies, Gillberg and Steffenburg ${ }^{12}$ followed up 46 young people of age between 16 and 23 years with infantile autism (diagnosed by DSM-III criteria) and "autistic-like conditions". Approximately one third developed epilepsy. There were two peaks of epilepsy: in infancy and around puberty, the majority being around puberty. Subsequent studies have yielded conflicting results, but there is some evidence, for example, the publication by Volkmar and Nelson, ${ }^{13}$ that the original conclusions of Gillberg and Steffenburg apply. Subsequent studies have yielded differing results for the age of onset of epilepsy in autism, as recently reviewed by El Achkar and Spence, ${ }^{14}$ and Bolton et al, ${ }^{15}$ in a long-term follow-up study of 150 individuals with childhood autism, found that $22 \%$ developed epilepsy; 
the majority were over 10 years of age and some developed epilepsy in adulthood.

\section{What is the influence of intellectual disability on the rate of epilepsy in children with autism?}

Amiet et al $^{1}$ carried out a meta-analysis on data available from published reports between 1963 and 2006 on autism and epilepsy to assess the relative risk of epilepsy in autism with respect to intellectual disability and gender. More subjects with autism who also had intellectual disability developed epilepsy than those who did not have an intellectual disability $(p<0.001)$. The pooled prevalence of epilepsy was $21.5 \%$ in those with autism and intellectual disability, compared with $8 \%$ in those with autism who did not have intellectual disability. They also noted that the male:female ratio of autism in those with epilepsy was close to $2: 1$, compared to a ratio of $3.5: 1$ in those without epilepsy. This analysis established very clearly that intellectual disability greatly increases the risk of epilepsy in people with autism. It also appeared to show that the rate of epilepsy in people with autism who did not have intellectual disability was higher, that is, $8 \%$, than in the general population, but this figure needs to be interpreted carefully. As already stated, it is highly likely that the IQ distribution of those who have autism but do not have intellectual disability is, nevertheless, shifted to the left, implying that the mean or median IQ would be considerably less than that of the general population and, consequently, the risk of epilepsy would be higher.

Other epidemiologic studies have confirmed an increased rate of autism in children with epilepsy. Davies et al, ${ }^{16}$ in a study based on the 1999 British Child and Adolescent Mental Health Survey, found that the rate of pervasive developmental disorder was $16 \%$ in those with "complicated epilepsy", which implied intellectual disability or other neurologic problems. Russ et $\mathrm{al}^{2}$ used data from the National Survey of Children's Mental Health 2007 in the USA and determined that the rate of ASD was $16 \%$ in those with epilepsy, compared with $1 \%$ in the general population, in children and young people $0-17$ years. Reilly et $\mathrm{al}^{3}$ studied children with epilepsy in Sussex schools and found a 21\% rate of ASD.

The prevalence of autism in people with epilepsy, in these and other studies, depends very much on the presence of intellectual disability and also on the definition of autism/ ASD used.

\section{Does epilepsy cause autism?}

In certain circumstances, for example, nonconvulsive status epilepticus and the Landau-Kleffner syndrome, ${ }^{17,18}$ epilepsy may cause features that have a strong resemblance to autism. The child may exhibit poor reciprocal social interaction, poor verbal and nonverbal communication and may cope with change poorly. However, this is not autism. Autism is a pervasive developmental disorder that persists over time. If either nonconvulsive status epilepticus or the LandauKleffner syndrome is treated effectively, the features of autism may decrease or even resolve. Would it be possible for nonconvulsive status epilepticus to continue, unabated, for several years, resulting in a clinical picture that was indistinguishable from autism? In theory, this possibility should be considered, although in practice, it is unlikely to occur. The models of the Landau-Kleffner syndrome and the closely allied continuous spike-waves in slow-wave sleep (CSWS) syndrome ${ }^{19}$ are particularly interesting. In the Landau-Kleffner syndrome, the child typically has normal early language development and then, apparently in association with electrical status epilepticus of slow-wave sleep (ESES), loses language skills. The pattern of loss of skills after normal early development also typically occurs in CSWS syndrome, but the loss of skills is not necessarily in the area of language. The typical case of the LandauKleffner syndrome would present with a verbal agnosia or a broader auditory agnosia (including the inability to recognize environmental sounds). Because the child cannot recognize his or her own speech, expressive language difficulties or even mutism can result. Children with the Landau-Kleffner syndrome can recover spontaneously with or without some permanent impairment of language or may continue to have severe language impairment. ${ }^{20}$ In cases in which there is no recovery, it is possible that the child could be misdiagnosed as having autism. Since $\sim 25 \%$ of children with autism appear to develop normally and then lose skills under 3 years of age, ${ }^{21}$ it would clearly be very important to distinguish between the loss of skills in autism, for which the cause remains unknown, and the loss of skills in the Landau-Kleffner syndrome, which is potentially treatable with recovery of skills if the treatment is instituted promptly. ${ }^{22}$

The examples of the Landau-Kleffner syndrome and the CSWS syndrome have led several researchers to ask the question: "Could loss of skills in classic autism be the result of epileptiform discharges or ESES?"23 It is said that approximately one-third of children with the LandauKleffner syndrome do not have obvious seizures before the onset of the syndrome, although they do have ESES. ${ }^{20} \mathrm{~A}$ particularly interesting study is that of Baird et al, ${ }^{24}$ who carried out an audit of sleep EEGs in 64 children with autism, none of whom had a history suggestive of epilepsy. 
Thirty-nine of the 64 children had autism with loss of skills and 20 of the total number had epileptiform abnormalities. The authors reported that there was no significant difference in the epileptiform activity in those who showed regression compared with those who did not. However, this was a relatively small study with a trend toward more epileptiform abnormalities in the children who regressed. In no case was ESES detected. Although the authors have not drawn particular attention to this point, in view of the trend, it is possible that a larger study might have shown a significant association between the degree of epileptiform abnormality and autistic regression. The other point that should be made is that there might be more than one cause of autistic regression; even if a small proportion of children with autistic regression have this as a result of epileptiform abnormality or ESES, the implication is that the regression in this subgroup might be halted or even reversed by appropriate antiepileptic treatment with medication or surgery. It might be argued that the weight of evidence is now against this hypothesis. The counterargument is that the importance of identifying the children whose apparent autistic regression might be the result of epilepsy/epileptiform activity, even if these are very few in number, can hardly be overemphasized since these children might be treatable with a return of at least a proportion of the lost skills. There is a case for carrying out a carefully conducted prospective study in all children who lose skills, within a given population, with thorough and appropriate investigation at the onset of the loss of skills; such investigation should include overnight EEG or at least sleep EEG recordings to exclude ESES or frequent epileptiform abnormalities.

In summary, the weight of the current evidence is against epilepsy being the cause of autism in the majority of cases. Whether there is a subgroup of children in whom undiagnosed epilepsy might have a role in causing autistic regression and in whom antiepileptic treatment might reverse the regression still remains undetermined, although attempts to establish such a link have not been successful so far.

While on one hand, it is important to treat the epilepsy early and effectively if it is suspected as being the cause of the autistic features, on the other hand, the epilepsy in most children will not be the cause of any autistic features. However, in both situations, good management of the epilepsy is strongly to be recommended because even if the epilepsy is not the cause of the autistic features, treating it effectively may improve the quality of the life of the individual and family greatly.

\section{Are specific epilepsy syndromes associated with autism?}

The behavioural aspects of pediatric epilepsy syndromes have been reviewed by the current author. ${ }^{25}$ Although several epilepsy syndromes are said to be associated with autistic features, in many cases, the diagnosis of autism has not been made according to rigorous criteria and the quality of the data is questionable.

Attention should be drawn to at least two syndromes in which autistic features are commonly reported. Reference has already been made to the Landau-Kleffner syndrome. The situation with West syndrome is particularly interesting. This syndrome is characterized by infantile spasms, hypsarrhythmia (a very disorganized high-voltage slowwave abnormality on the EEG) and loss of skills. There is a high rate of autism in children who have West syndrome as a result of the genetic disorder, tuberous sclerosis. There is also an increased rate of autism in children with West syndrome/infantile spasms that is either idiopathic or due to other genetic conditions. ${ }^{26}$ Bolton et $\mathrm{al}^{27}$ showed that autism in tuberous sclerosis was associated with a history of West syndrome and temporal tubers. It is not surprising that an epilepsy syndrome that is associated with such a marked loss of skills should also be associated with autism. The finding that the autism was particularly prevalent in children with tuberous sclerosis who also had temporal tubers is of added interest. The loss of skills in West syndrome is associated with the gross EEG abnormality of hypsarrhythmia. Children with West syndrome lose skills permanently in association with this EEG disturbance, suggesting that if treatment could be given soon enough to stop the epileptiform abnormality, the children might deteriorate less or might even regain skills. The study by Jambaque et $\mathrm{al}^{28}$ appeared to confirm this when children with tuberous sclerosis and West syndrome were treated with vigabatrin and had an increase in mental age. This again emphasizes the importance of prompt effective treatment in cases where loss of skills might be the result of epilepsy or epileptiform disturbance as demonstrated by a gross EEG abnormality.

\section{Can autism cause epilepsy?}

From the available evidence, it appears that epilepsy is rarely the cause of autistic features, although when it is, it is important to treat it promptly and effectively. There is no plausible explanation for a link in the other direction; in other words, there is no plausible argument for suggesting that autism could cause epilepsy. It seems that the weight of 
evidence now favors a common underlying cause for both conditions. Broadly speaking, such underlying causes could be divided into genetic and environmental.

\section{What is the role of genetics in determining conditions that underlie both epilepsy and autism?}

There have been remarkable advances in genetics over recent years. With regard to autism, these advances are epitomized in the title of the paper by Betancur, ${ }^{29}$ "Etiological heterogeneity in autism spectrum disorders: more than 100 genetic and genomic disorders and still counting". Although not all of these disorders would necessarily have been associated with epilepsy, many of them would have been. Intellectual disability is strongly associated with epilepsy. The lower the intellectual ability, the higher the prevalence of both epilepsy and autism. ${ }^{30-33}$ Much attention has rightly been given to the obvious example of a genetic disorder that can result in both autism and epilepsy, namely, tuberous sclerosis. ${ }^{27}$ In this particular case, a new type of treatment, namely, mTOR inhibitors such as sirolimus (rapamycin, Rapamune, Pfizer) and everolimus (Afinitor, Novartis) that limit cell growth, can also have an effect in ameliorating seizures, in at least some cases. ${ }^{34,35}$

Advances in chromosomal microarray analysis have made detection of small chromosomal variations possible. In the past, clinicians would examine the patient, perhaps with dysmorphic features, intellectual disability, autism and epilepsy, expressing the view that a chromosomal abnormality was highly likely, only to find that chromosomal investigations revealed no such abnormality. The reverse is now true. Chromosomal microanalysis often yields unsuspected abnormalities, the significance of which may be difficult to interpret. ${ }^{36}$

Among the genetic abnormalities that have been identified as leading both to autism and epilepsy, in at least some cases, there have been a number of copy number variants. Some of the abnormalities identified have included 1q21 deletions, 7q11.23 deletions, 15q11.1-q13.3 duplications, 16p11.2 deletions, 7q11.23 duplications, 18q12.1 duplications and $22 \mathrm{q} 11.2$ deletions. Lee et $\mathrm{al}^{37}$ recently reviewed the genetic abnormalities associated with ASD and epilepsy. Blackmon ${ }^{38}$ has reviewed the genetic and chromosomal abnormalities associated with brain malformations that can lead to both conditions. A number of studies have found abnormalities in corpus callosum connectivity associated with ASD, as pointed out recently by Margari et al. ${ }^{39}$ They stated that about $20 \%$ of corpus callosum abnormalities are caused by single or multiple gene mutations or by chromosomal abnormalities. In their own study of 61 patients with corpus callosum abnormalities, 4 had ASD and 36 had epilepsy.

An interesting population-based, nationwide cohort study was carried out in Sweden by Sundelin et al. ${ }^{40}$ The study design was unusual. They examined follow-up data for 85,201 individuals with epilepsy, 80,511 siblings of these individuals and 98,534 offspring. In addition, those with epilepsy were compared with five controls, matched for age, sex, calendar period and county. Siblings and offspring were compared with siblings and offspring of controls. They excluded siblings and offspring with epilepsy. Because they were only interested in those who developed ASD, the percentages do not represent an overall prevalence, but do provide a relative a risk. During the follow-up period, 1,381 $(1.6 \%)$ of those with epilepsy and $700(0.2 \%)$ controls were diagnosed with ASD. The hazard ratio for developing ASD in those with epilepsy was 10.49, and 95\% CI was 9.55-11.53. The highest risk was in those who had epilepsy diagnosed in childhood. It was of interest to note that both siblings and offspring of epilepsy patients had an increased risk of developing ASD, suggesting that both epilepsy and ASD had "shared etiology". This is likely to be interpreted as a shared genetic risk, although environmental factors might also play a role. A commentary on this study was provided by the current author. ${ }^{41}$

There are many genetically determined metabolic conditions that lead to intellectual disability and the combination of both autism and epilepsy. The classic example is phenylketonuria, ${ }^{42}$ the deleterious effects of which have largely been eliminated in developed countries by neonatal screening.

\section{Environmental factors}

Schmidt et $\mathrm{al}^{43}$ have reviewed the environmental factors associated with autism, some of which may also be associated with epilepsy. There is evidence for a deleterious effect of air pollution. There is less convincing evidence for the role of organophosphate pesticides and phthalate exposure. They have pointed to evidence for a protective environmental effect of maternal folate during pregnancy. There is an increasing recognition of gene-environment interactions in general. If the offspring has a genetic predisposition and the mother is exposed to a relevant environmental toxin, the combination might result in a child with ASD who could also have epilepsy. Much further work needs to be performed in this area to provide definitive answers. 
A more obvious environmental link is that of intrauterine infection. For example, maternal rubella during pregnancy has long been associated with a high risk of intellectual disability, autism and epilepsy in the offspring. ${ }^{44}$ If the mother takes the antiepileptic drug sodium valproate during pregnancy, this can also affect the brain development of the fetus, leading to intellectual disability and autism. ${ }^{45}$ Christensen et $\mathrm{al}^{46}$ have reviewed the role of maternal valproate. This is an interesting situation in which epilepsy in the mother can, through the environmental effect of the medication she takes, predispose to autism in the offspring.

Brain damage arising during delivery and through neonatal factors such as severe neonatal jaundice should be avoidable, but if treated inadequately can predispose both to epilepsy and autism. In a recent large $(\mathrm{N}=874), 10$-year follow-up study of children born under 28 weeks gestation, Kuban et $\mathrm{a}^{47}$ reported a high ASD rate: $9 \%$ in boys and $5 \%$ in girls. Singh et al ${ }^{48}$ have recently reviewed the shared features of the effects of traumatic brain injury and autism.

There are many metabolic conditions that can lead to brain damage if inadequately treated. The total number of these that could lead to intellectual disability, epilepsy and autism, especially if inadequately treated, remains unclear. ${ }^{49}$ However, Frye ${ }^{49}$ has provided a strong argument for treating any underlying metabolic disorders, both for ameliorating autism and epilepsy. Some studies have suggested that autistic features could be improved by appropriate treatment of the metabolic disorder. This raises an important question that continues to be debated, namely, how extensively should a child with autism and loss of skills be investigated? Again, it should be emphasized that the greater the level of intellectual disability, the higher the probability of epilepsy developing.

\section{How should the individual with both epilepsy and autism be managed?}

As a general principle, both conditions should be managed as they would be when occurring individually, but with certain reservations. This management will include ensuring that the cause of the epilepsy is appropriately investigated.

First, as has already been emphasized, ensure that the autistic features are not the result of ESES or frequent epileptiform discharges.

The diagnosis of epilepsy may be more difficult in someone with autism, especially if there is accompanying intellectual disability. Autism alone or in combination with intellectual disability can prove to be an obstacle in history taking.
There are special cases, for example, tuberous sclerosis, where specific treatment - in this case with sirolimus (rapamycin) or everolimus - may decrease the seizures (see earlier). ${ }^{50}$

Some antiepileptic drugs can have negative effects on mood, behavior or cognition. For example, levetiracetam is sometimes associated with deterioration in children, whereas lamotrigine tends to be a mood-leveling antiepileptic drug. Topiramate can be associated with word-finding difficulties. For more details, see recent reviews by Aldenkamp et $\mathrm{al}^{51}$ and Brodie et al..$^{52}$

One of the most dramatic developments in recent years in the field of neurology has been the discovery of the role of neuronal autoantibodies, which can result in seizures, loss of skills, which can sometimes be dramatic, behavioral changes and even psychosis. Prompt, effective immunotherapy can, in at least some cases, reverse all these changes. ${ }^{53,54}$ Very few studies consider the possibility that neuronal antibodies might be a cause for at least some cases of autistic regression. ${ }^{55}$ This is an area that requires further consideration.

\section{How should additional comorbidities be treated in the presence of both epilepsy and autism?}

In addition to intellectual disability, which has already been discussed, three common comorbidities with autism and epilepsy are ADHD, anxiety and sleep problems.

\section{Attention deficit hyperactivity disorder}

ADHD is common in children with epilepsy, children with autism and children with both conditions together. ${ }^{7}$ Should children with autism who have both epilepsy and ADHD be considered for ADHD treatment? The answer to this question depends on the circumstances. Some children with epilepsy present with features of ADHD that are the result of frequent epileptiform discharges. In those cases, treating the epileptiform discharges may improve the ADHD features and this would be the treatment of choice. A few children may have inattention, distractibility and excitability as a result of inappropriate antiepileptic medication; for example, treatment with phenobarbital, benzodiazepines or vigabatrin can result in this situation. In those cases, a review of the antiepileptic medication and treatment with an antiepileptic drug that does not cause these features would be appropriate. However, many children with epilepsy and autism appear to have genuine underlying ADHD. In those cases, standard treatment with ADHD medication can be of great benefit. There are several research papers and reviews that have come 
to the conclusion that ADHD treatment is very unlikely to precipitate seizures. The current author has reviewed this topic elsewhere. ${ }^{56}$ One of the most convincing studies is that of Gucuyener et al, ${ }^{57}$ who treated 57 children with ADHD and active seizures and 62 children with ADHD and EEG abnormalities. They concluded that the ADHD symptoms improved significantly, but seizure frequency did not change from baseline. Methylphenidate appeared to have a beneficial effect on the EEG. They concluded that methylphenidate is safe and effective in children with ADHD and concomitant active seizures or EEG abnormalities. When endeavoring to assess the effect of ADHD medication on seizures, there are at least two confounding factors. First, epilepsy is a highly variable condition. If no change is made to treatment, some subjects will have an increase in seizure frequency, some will have a decrease and others will have no change, if the seizure frequency is monitored over a relatively short period of time. If ADHD medication is instituted, it may consequently be incorrectly concluded that those who had an increase in seizures did so because of the ADHD treatment, whereas, in fact, it was simply a variation in the epilepsy that would have been expected in any case. A different situation is that of the child who has no history of seizures, but develops seizures while taking ADHD medication. Can it be concluded that the ADHD medication caused the seizure? Hemmer et $\mathrm{al}^{58}$ investigated 234 children with "uncomplicated ADHD" who had no history of seizures. Thirty-six (15.4\%) had epileptiform abnormalities. Studies on children with ADHD consistently reveal a high proportion of epileptiform abnormalities, implying that ADHD itself is a risk factor for having seizures. Consequently, even if a child with ADHD has no previous history of seizures and develops seizures after ADHD medication is commenced, it cannot necessarily be concluded that the ADHD medication was the cause of the seizures; ADHD itself predisposes to seizures.

Are there any additional factors that should be taken into account when considering methylphenidate treatment for a child with autism, epilepsy and ADHD? Methylphenidate can sometimes increase "obsessionality", with the implication that some of the rituals in a child with autism may become more intrusive. In the experience of the current author, the advantages of ADHD treatment usually outweigh this disadvantage; but occasionally, the "obsessionality" can become so intrusive that it is necessary to review the ADHD medication and to prescribe an alternative to methylphenidate.

In children with autism, epilepsy and ADHD, there is relatively little evidence on other ADHD treatments, such as atomoxetine, guanfacine and clonidine. However, there seems to be no clear evidence that these medications exacerbate seizures either. The current author has used atomoxetine and clonidine in children with autism, uncontrolled epilepsy and ADHD, without the occurrence of any seizure exacerbations.

In summary, the evidence reveals that about $30 \%$ of children with epilepsy have ADHD and about $70 \%$ of those with epilepsy and ADHD will benefit from treatment of the ADHD with methylphenidate. ${ }^{56}$ There has been a suggestion that the proportion might be slightly smaller in those who also have autism, but the proportion that responds favorably to standard ADHD medication is, nevertheless, high. Children with epilepsy and autism who also have ADHD should not be denied treatment for the ADHD, which can be of great benefit to them.

\section{Anxiety}

Anxiety is common in young people who have both epilepsy and autism. ${ }^{59}$ The RUPP study has shown that behavior in children with autism and intellectual disability can be improved with low-dose risperidone. ${ }^{60}$ The mechanism is probably through decreasing anxiety. Is epilepsy a contraindication to such treatment? Although there are anecdotal reports of seizure exacerbations with risperidone, it appears that lowdose risperidone is very unlikely to exacerbate seizures.$^{61}$ As with many medications, the risk almost certainly increases with increasing dose; but since the doses required for treating anxiety in children with autism are low, the chances of seizure exacerbations are also low, even in children with uncontrolled epilepsy and autism. However, it is important to be aware of the interactions between antiepileptic and antipsychotic drugs. In particular, enzyme-inducing drugs such as carbamazepine, phenobarbital and phenytoin may decrease the blood levels of antipsychotic medication. For example, carbamazepine decreases the serum levels of both risperidone and its active metabolite. ${ }^{62}$ This implies that larger doses of risperidone may be required in those who are also taking carbamazepine or possibly other enzyme-inducing AEDs.

Cognitive-behavioral therapy and other psychologic interventions should be the first-line approach to managing anxiety. Although selective serotonin reuptake inhibitors are generally effective in decreasing anxiety in adults and teenagers, a Cochrane review has concluded that there is a lack of evidence for a beneficial role of selective serotonin reuptake inhibitors in treating anxiety in children with autism.

\section{Sleep}

Sleep problems are very common in children with autism, with or without epilepsy. ${ }^{63,64}$ If sleep problems present in a 
child with both epilepsy and autism, it is clearly important to ensure that nocturnal seizures are not the reason for the sleep disturbance. If they are, a review of antiepileptic medication is required. If the sleep disturbance is not due to nocturnal seizures, is melatonin a contraindication? The current author reviewed the question of whether melatonin exacerbates seizures. ${ }^{65}$ Although the literature is sparse, there appears to be no good evidence that melatonin exacerbates seizures. The current author has used melatonin in many children with uncontrolled epilepsy, with no reports of seizure exacerbations. Animal work suggests that melatonin might have an antiepileptic effect. ${ }^{66,67}$ Based on the current, albeit incomplete, evidence, there seems to be no reason for avoiding melatonin as a treatment for sleep-onset insomnia in children with epilepsy and autism.

\section{Future research}

Based on the limited evidence that is available in many areas of research on epilepsy and autism, the following recommendations may be made.

With regard to autism with regression, there is a pressing need for carefully conducted, prospective, population-based studies particularly to investigate the child as soon as any regression is detected. Such investigation should include the following:

1. Genetic and chromosomal studies

2. Search for metabolic and mitochondrial disorders

3. EEGs including at least a sleep EEG and preferably an overnight EEG

4. Testing for possible neuronal antibodies.

With regard to autism in general, in addition to the first two investigations listed above, a search should be made for possible environmental factors, including prenatal factors such as maternal exposure to infection, pollution, toxic chemicals, alcohol and drugs, both illicit and therapeutic. The history of drug exposure in mothers who also have epilepsy should include a history of exposure to antiepileptic drugs in view of the link between maternal valproate and learning problems/probable autism in the offspring.

Obstetric and neonatal factors will form an essential part of the data.

As stratification into the various subcategories is likely to result in small numbers, there is a strong case for international research collaboration with agreed and standardized protocols. The aim will be that focused research studies provide answers that significantly improve the future outlook for people with epilepsy and autism.

\section{Conclusion}

1. The risk of epilepsy in people with autism is high, around $20 \%$ based on previous criteria of autism, and the risk of ASD in epilepsy is also high, probably around $20 \%$, based on recent epidemiologic studies.

2. Although, in most cases, the link between epilepsy and autism arises from a common underlying factor, it is essential to ensure that the autistic features are not the result of inadequately treated epilepsy.

3. Good management of autism, epilepsy and comorbidities can greatly improve the quality of life of both the child and the family.

There are major challenges both in the future research and in the current management of people who have both epilepsy and autism. However, prompt, appropriate management, with a good understanding of the importance of focused investigations and correct treatment, can be of significant benefit.

\section{Disclosure}

The author reports no conflicts of interest in this work.

\section{References}

1. Amiet C, Gourfinkel-An I, Bouzamondo A, et al. Epilepsy in autism is associated with intellectual disability and gender: evidence from a meta-analysis. Biol Psychiatry. 2008;64(7):577-582.

2. Russ SA, Larson K, Halfon N. A national profile of childhood epilepsy and seizure disorder. Pediatrics. 2012;129(2):256-264.

3. Reilly C, Atkinson P, Das KB, et al. Neurobehavioral comorbidities in children with active epilepsy: a population-based study. Pediatrics. 2014;133(6):e1586-e1593.

4. Kanner L. Autistic disturbances of affective contact. Nerv Child. 1943; 2(3):217-250.

5. Tuchman R, Rapin I. Epilepsy in autism. Lancet Neurol. 2002;1(6): 352-358.

6. Besag FM. Current controversies in the relationships between autism and epilepsy. Epilepsy Behav. 2015;47:143-146.

7. Sillanpää M, Besag F, Aldenkamp A, Caplan R, Dunn DW, Gobbi G. Psychiatric and behavioural disorders in children with epilepsy (ILAE Task Force Report): epidemiology of psychiatric/behavioural disorder in children with epilepsy. Epileptic Disord. 2016;18(s1):S2-S7.

8. Lotter V. Epidemiology of autistic conditions in young children. Soc Psychiatry Psychiatric Epidemiol. 1966;1(3):124-135.

9. Baird G, Simonoff E, Pickles A, et al. Prevalence of disorders of the autism spectrum in a population cohort of children in South Thames: the Special Needs and Autism Project (SNAP). Lancet. 2006; 368(9531):210-215.

10. Fombonne E. Estimated prevalence of autism spectrum conditions in Cambridgeshire is over 1\%. Evidence Based Mental Health. 2010; 13(1):32.

11. Kim YS, Fombonne E, Koh YJ, Kim SJ, Cheon KA, Leventhal BL. A comparison of DSM-IV pervasive developmental disorder and DSM-5 autism spectrum disorder prevalence in an epidemiologic sample. J Am Acad Child Adolesc Psychiatry. 2014;53(5):500-508.

12. Gillberg C, Steffenburg S. Outcome and prognostic factors in infantile autism and similar conditions: a population-based study of 46 cases followed through puberty. J Autism Dev Disord. 1987;17(2):273-287.

13. Volkmar FR, Nelson DS. Seizure disorders in autism. J Am Acad Child Adolesc Psychiatry. 1990;29(1):127-129. 
14. El Achkar CM, Spence SJ. Clinical characteristics of children and young adults with co-occurring autism spectrum disorder and epilepsy. Epilepsy Behav. 2015;47:183-190.

15. Bolton PF, Carcani-Rathwell I, Hutton J, Goode S, Howlin P, Rutter M. Epilepsy in autism: features and correlates. Br J Psychiatry. 2011;198(4): 289-294.

16. Davies S, Heyman I, Goodman R. A population survey of mental health problems in children with epilepsy. Dev Med Child Neurol. 2003;45(5): 292-295.

17. Landau WM, Kleffner FR. Syndrome of acquired aphasia with convulsive disorder in children. Neurology. 1957;7:523-530.

18. Deonna TW. Acquired epileptiform aphasia in children (LandauKleffner syndrome). J Neurophysiol. 1991;8(3):288-298.

19. Roulet Perez E, Davidoff V, Despland PA, Deonna T. Mental and behavioural deterioration of children with epilepsy and CSWS: acquired epileptic frontal syndrome. Dev Med Child Neurol. 1993;35(8): 661-674.

20. Deonna T, Beaumanoir A, Gaillard F, Assal G. Acquired aphasia in childhood with seizure disorder: a heterogeneous syndrome. Neuropadiatrie. 1977;8(3):263-273.

21. Parr JR, Le Couteur A, Baird G, et al. Early developmental regression in autism spectrum disorder: evidence from an international multiplex sample. J Autism Dev Disord. 2011;41(3):332-340.

22. Irwin K, Birch V, Lees J, et al. Multiple subpial transection in LandauKleffner syndrome. Dev Med Child Neurol. 2001;43(4):248-252.

23. Deonna T, Roulet E. Autistic spectrum disorder: evaluating a possible contributing or causal role of epilepsy. Epilepsia. 2006;47 (Suppl 2): 79-82.

24. Baird G, Robinson RO, Boyd S, Charman T. Sleep electroencephalograms in young children with autism with and without regression. Dev Med Child Neurol. 2006;48(7):604-608.

25. Besag FMC. Behavioral aspects of pediatric epilepsy syndromes. Epilepsy Behav. 2004;5 (Suppl 1):S3-S13.

26. Pavone P, Striano P, Falsaperla R, Pavone L, Ruggieri M. Infantile spasms syndrome, West syndrome and related phenotypes: what we know in 2013. Brain Dev. 2014;36(9):739-751.

27. Bolton PF, Park RJ, Higgins JN, Griffiths PD, Pickles A. Neuro-epileptic determinants of autism spectrum disorders in tuberous sclerosis complex. Brain. 2002;125(Pt 6):1247-1255

28. Jambaque I, Mottron L, Chiron C. Neuropsychological outcome in children with West syndrome. In: Jambaque I, Lassonde M, Dulac O, editors. Neuropsychology of Childhood Epilepsy. New York: Kluwer Academic/Plenum Publishers; 2001:175-183.

29. Betancur C. Etiological heterogeneity in autism spectrum disorders: more than 100 genetic and genomic disorders and still counting. Brain Res. 2011;1380:42-77.

30. Szatmari P, Jones MB. IQ and the genetics of autism. J Child Psychol Psychiatry. 1991;32(6):897-908.

31. Charman T, Pickles A, Simonoff E, Chandler S, Loucas T, Baird G. IQ in children with autism spectrum disorders: data from the Special Needs and Autism Project (SNAP). Psychol Med. 2011;41(3):619-627.

32. Forsgren L, Edvinsson SO, Blomquist HK, Heijbel J, Sidenvall R. Epilepsy in a population of mentally retarded children and adults. Epilepsy Res. 1990;6(3):234-248.

33. Steffenburg U, Hagberg G, Viggedal G, Kyllerman M. Active epilepsy in mentally retarded children. I. Prevalence and additional neuroimpairments. Acta Paediatri. 1995;84(10):1147-1152.

34. Canpolat M, Per H, Gumus H, et al. Rapamycin has a beneficial effect on controlling epilepsy in children with tuberous sclerosis complex: results of 7 children from a cohort of 86. Childs Nerv Syst. 2014; 30(2):227-240.

35. French JA, Lawson JA, Yapici Z, et al. Adjunctive everolimus therapy for treatment-resistant focal-onset seizures associated with tuberous sclerosis (EXIST-3): a phase 3, randomised, double-blind, placebocontrolled study. Lancet. 2016;388(10056):2153-2163.

36. Paciorkowski AR, Fang M. Chromosomal microarray interpretation: what is a child neurologist to do? Pediatr Neurol. 2009;41(6):391-398.
37. Lee BH, Smith T, Paciorkowski AR. Autism spectrum disorder and epilepsy: disorders with a shared biology. Epilepsy Behav. 2015;47: 191-201.

38. Blackmon K. Structural MRI biomarkers of shared pathogenesis in autism spectrum disorder and epilepsy. Epilepsy Behav. 2015;47: $172-182$.

39. Margari L, Palumbi R, Campa MG, et al. Clinical manifestations in children and adolescents with corpus callosum abnormalities. J Neurol. 2016;263(10):1939-1945.

40. Sundelin HE, Larsson H, Lichtenstein P, et al. Autism and epilepsy: a population-based nationwide cohort study. Neurology. 2016;87(2): 192-197.

41. Besag FM. Rate of epilepsy in people with autism and the rate of autism in people with epilepsy are high. Evid Based Med. 2016;21(6):230.

42. Baieli S, Pavone L, Meli C, Fiumara A, Coleman M. Autism and phenylketonuria. J Autism Dev Disord. 2003;33(2):201-204.

43. Schmidt RJ, Lyall K, Hertz-Picciotto I. Environment and autism: current state of the science. Cut Edge Psychiatry Pract. 2014;1(4):21-38.

44. Chess S. Follow-up report on autism in congenital rubella. J Autism Child Schizophr. 1977;7(1):69-81.

45. Meador KJ, Loring DW. Prenatal valproate exposure is associated with autism spectrum disorder and childhood autism. J Pediatr. 2013; 163(3):924.

46. Christensen J, Gronborg TK, Sorensen MJ, et al. Prenatal valproate exposure and risk of autism spectrum disorders and childhood autism. JAMA. 2013;309(16):1696-1703.

47. Kuban KC, Joseph RM, O'shea TM, et al. Girls and boys born before 28 weeks gestation: risks of cognitive, behavioral, and neurologic outcomes at age 10 years. J Pediatr. 2016;173:69-75. e61.

48. Singh R, Turner RC, Nguyen L, Motwani K, Swatek M, Lucke-Wold BP. Pediatric traumatic brain injury and autism: elucidating shared mechanisms. Behav Neurol. 2016;2016:8781725.

49. Frye RE. Metabolic and mitochondrial disorders associated with epilepsy in children with autism spectrum disorder. Epilepsy Behav. 2015;47: $147-157$.

50. Krueger DA, Northrup H, Group ITSCC. Tuberous sclerosis complex surveillance and management: recommendations of the 2012 International Tuberous Sclerosis Complex Consensus Conference. Pediatr Neurol. 2013;49(4):255-265.

51. Aldenkamp A, Besag F, Gobbi G, Caplan R, Dunn DW, Sillanpää M. Psychiatric and behavioural disorders in children with epilepsy (ILAE Task Force Report): adverse cognitive and behavioural effects of antiepileptic drugs in children. Epileptic Disord. 2016;18(s1): S55-S67.

52. Brodie MJ, Besag F, Ettinger AB, et al. Epilepsy, antiepileptic drugs, and aggression: an evidence-based review. Pharmacol Rev. 2016;68(3): 563-602.

53. Vincent A, Bien CG, Irani SR, Waters P. Autoantibodies associated with diseases of the CNS: new developments and future challenges. Lancet Neurol. 2011;10(8):759-772.

54. Irani SR, Gelfand JM, Al-Diwani A, Vincent A. Cell-surface central nervous system autoantibodies: clinical relevance and emerging paradigms. Ann Neurol. 2014;76(2):168-184.

55. Dalton $\mathrm{P}$, Deacon R, Blamire A, et al. Maternal neuronal antibodies associated with autism and a language disorder. Ann Neurol. 2003;53(4): 533-537.

56. Besag F, Gobbi G, Caplan R, Sillanpää M, Aldenkamp A, Dunn DW. Psychiatric and behavioural disorders in children with epilepsy (ILAE Task Force Report): epilepsy and ADHD. Epileptic Disord. 2016;18(s1):S8-S15.

57. Gucuyener K, Erdemoglu AK, Senol S, Serdaroglu A, Soysal S, Kockar AI. Use of methylphenidate for attention-deficit hyperactivity disorder in patients with epilepsy or electroencephalographic abnormalities. J Child Neurol. 2003;18(2):109-112.

58. Hemmer SA, Pasternak JF, Zecker SG, Trommer BL. Stimulant therapy and seizure risk in children with ADHD. Pediatr Neurol. 2001;24(2): 99-102. 
59. Dunn DW, Besag F, Caplan R, Aldenkamp A, Gobbi G, Sillanpää M. Psychiatric and behavioural disorders in children with epilepsy (ILAE Task Force Report): anxiety, depression and childhood epilepsy. Epileptic Disord. 2016;18(s1):S24-S30.

60. McDougle CJ, Scahill L, Aman MG, et al. Risperidone for the core symptom domains of autism: results from the study by the autism network of the research units on pediatric psychopharmacology. Am J Psychiatry. 2005;162(6):1142-1148.

61. Holzhausen SP, Guerreiro MM, Baccin CE, Montenegro MA. Use of risperidone in children with epilepsy. Epilepsy Behav. 2007;10(3): $412-416$.

62. Spina E, Avenoso A, Facciola G, et al. Plasma concentrations of risperidone and 9-hydroxyrisperidone: effect of comedication with carbamazepine or valproate. Ther Drug Monit. 2000;22(4):481-485.
63. Gail Williams P, Sears LL, Allard A. Sleep problems in children with autism. J Sleep Res. 2004;13(3):265-268.

64. Malow BA. Sleep disorders, epilepsy, and autism. Ment Retard Dev Disabil Res Rev. 2004;10(2):122-125.

65. Jain S, Besag FM. Does melatonin affect epileptic seizures? Drug Saf. 2013;36(4):207-215.

66. Lima E, Cabral FR, Cavalheiro EA, Naffah-Mazzacoratti MG, Amado D. Melatonin administration after pilocarpine-induced status epilepticus: a new way to prevent or attenuate postlesion epilepsy? Epilepsy Behav. 2011;20(4):607-612.

67. Solmaz I, Gurkanlar D, Gokcil Z, Goksoy C, Ozkan M, Erdogan E. Antiepileptic activity of melatonin in guinea pigs with pentylenetetrazolinduced seizures. Neurol Res. 2009;31(9):989-995.

\section{Publish your work in this journal}

Neuropsychiatric Disease and Treatment is an international, peerreviewed journal of clinical therapeutics and pharmacology focusing on concise rapid reporting of clinical or pre-clinical studies on a range of neuropsychiatric and neurological disorders. This journal is indexed on PubMed Central, the 'PsycINFO' database and CAS, and is the official journal of The International Neuropsychiatric Association (INA). The manuscript management system is completely online and includes a very quick and fair peer-review system, which is all easy to use. Visit http://www.dovepress.com/testimonials.php to read real quotes from published authors.

Submit your manuscript here: http://www.dovepress.com/neuropsychiatric-disease-and-treatment-journal 